\title{
Schur convexity of Bonferroni harmonic mean
}

\author{
Sreenivasa Reddy Perla ${ }^{1}$ (D) . S. Padmanabhan ${ }^{2}$
}

Received: 31 January 2018 / Accepted: 7 June 2018 / Published online: 16 July 2018

(c) The Author(s) 2018

\begin{abstract}
In this paper, we research the Schur convexity, Schur geometric convexity and Schur harmonic convexity of the Bonferroni harmonic mean. Some inequalities identified with the Bonferroni harmonic mean are set up to represent the utilizations of the acquired outcomes.
\end{abstract}

Keywords Bonferroni harmonic mean · Schur's condition · Majorization relationship · Inequality

Mathematics Subject Classification Prime 4600

\section{Introduction}

Arithmetic, Geometric and Harmonic means are three important means, which have been extensively used in the information aggregation $[5,6,7,11,12,17,18,19,35$, 36]. For a collection of real numbers $a_{i}(i=1,2, \ldots n)$, the Arithmetic mean (AM), the Geometric mean (GM) and the Harmonic mean (HM) are defined by:

$$
\begin{aligned}
& \operatorname{AM}_{i}(i=1,2 \ldots n)=\frac{1}{n} \sum_{i=1}^{n} a_{i} \\
& \operatorname{BM} a_{i}(i=1,2 \ldots n)=\frac{1}{n} \prod_{i=1}^{n} a_{i}^{n}
\end{aligned}
$$

Sreenivasa Reddy Perla

srireddy_sri@yahoo.co.in

S. Padmanabhan

padmanabhanrnsit@gmail.com

1 Department of Mathematics, The Oxford College of Engineering, Bengaluru, India

2 Department of Mathematics, R N S I T, Uttarahalli-Kengeri Road, Bengaluru 560 098, India 


$$
\operatorname{HM} a_{i}(i=1,2 \ldots n)=n / \sum_{i=1}^{n} \frac{1}{a_{i}}
$$

respectively. The fundamental characteristic of arithmetic mean is that it focuses on the group opinions, while geometric mean gives more importance to the individual opinions and harmonic mean is the reciprocal of arithmetic mean, which is a conservative average to be used to provide for aggregation lying between the max and min operators and is widely used as a tool to aggregate central tendency data [30].

In the existing literature, the harmonic mean is generally considered as a fusion technique of numerical data information. However, in many situations the input arguments take the form of triangular fuzzy numbers because of time pressure, lack of knowledge, and peoples limited expertise related with problem domain. Therefore, "how to aggregate fuzzy data by using the harmonic mean?" is an interesting research topic and is worth paying attention too. So Xu [30] developed the fuzzy harmonic mean operators such as fuzzy weighted harmonic mean (FWHM) operator, fuzzy ordered weighted harmonic mean (FOWHM) operator and fuzzy hybrid harmonic mean (FHHM) operator and applied them to MAGDM. Wei [25] developed fuzzy induced ordered weighted harmonic mean (FIOWHM) operator and then based on the FWHM and FIOWHM operators, presented the approach to MAGDM. H. Sun and M. Sun [23] further applied the BM operator to fuzzy environment, introduced the fuzzy Bonferroni harmonic mean (FBHM) operator and the fuzzy ordered Bonferroni harmonic mean (FOBHM) operator and applied the FOBHM operator to multiple attribute decision making.

The Bonferroni mean operator was initially proposed by Bonferroni [2] and was also investigated intensively by Yager [32].

Definition 1.1 [2] Let $\mathrm{p}, q>0, p+q \neq 0$ and let $a_{i}(i=1,2, \ldots n)$ be a collection of non-negative numbers. If

$$
\mathrm{BM}^{p, q}\left(a_{1}, a_{2}, \ldots a_{n}\right)=\left(\frac{1}{n(n-1)} \sum_{i, j=1, i \neq j}^{n} x_{i}^{p} x_{j}^{q}\right)^{\frac{1}{p+q}}
$$

then $\mathrm{BM}^{p, q}$ is called the Bonferroni mean $(\mathrm{BM})$ operator. It has important application in multi criteria decision-making $[1,13,14,29,31,32]$.

Beliakov et al. [1] further extended the BM operator by considering the correlations of any three aggregated arguments instead of any two.

Definition 1.2 [1] Let $\mathrm{p}, q, r>0, p+q+r \neq 0$ and let $a_{i}(i=1,2, \ldots n)$ be a collection of non-negative numbers. If

$$
\operatorname{GBM}^{p, q, r}\left(a_{1}, a_{2}, \ldots a_{n}\right)=\left(\frac{1}{n(n-1)(n-2)} \sum_{i, j, k=1, i \neq j \neq k}^{n} x_{i}^{p} x_{j}^{q} x_{k}^{r}\right)^{\frac{1}{p+q+r}}
$$


then $\mathrm{GBM}^{p, q, r}$ is called the generalized Bonferroni mean (GBM) operator. In particular, if $r=0$, then the GBM operator reduces to the BM operator. However, it is noted that both BM operator and the GBM operator do not consider the situation that $i=j$ or $j=k$ or $i=k$, and the weight vector of the aggregated arguments is not also considered. To overcome this drawback, Xia et al. [29]. defined the weighted version of the GBM operator.

Definition 1.3 [29] Let p, $q, r>0, p+q+r \neq 0$ and let $a_{i}(i=1,2, \ldots n)$ be a collection of non-negative numbers with the weight vector $w=\left(w_{1}, w_{2}, \ldots w_{n}\right)^{T}$ such that $w_{i} \geq 0, i=1,2, \ldots n$ and $\sum_{i=1}^{n} w_{i}=1$. If

$$
\operatorname{GWBM}^{p, q, r}\left(a_{1}, a_{2}, \ldots a_{n}\right)=\left(\sum_{i, j, k=1, i \neq j \neq k}^{n} w_{i} w_{j} w_{k} x_{i}^{p} x_{j}^{q} x_{k}^{r}\right)^{\frac{1}{p+q+r}}
$$

then $\mathrm{GWBM}^{p, q, r}$ is called the generalized weighted Bonferroni mean (GWBM) operator.

Some special cases can be obtained as the change of the parameters as follows:

Case 1 If $r=0$ then the GWBM operator reduces to the following:

$$
\begin{aligned}
\operatorname{GWBM}^{p, q, 0}\left(a_{1}, a_{2}, \ldots a_{n}\right) & =\left(\sum_{i, j, k=1, i \neq j \neq k}^{n} w_{i} w_{j} w_{k} x_{i}^{p} x_{j}^{q}\right)^{\frac{1}{p+q}} \\
& =\left(\sum_{i, j=1, i \neq j}^{n} w_{i} w_{j} x_{i}^{p} x_{j}^{q} \sum_{k=1}^{n} w_{k}\right)^{\frac{1}{p+q}} \\
\operatorname{GWBM}^{p, q, 0}\left(a_{1}, a_{2}, \ldots a_{n}\right) & =\left(\sum_{i=1}^{n} w_{i} w_{j} x_{i}^{p} x_{j}^{q}\right)^{\frac{1}{p+q}}
\end{aligned}
$$

which is the weighted Bonferroni mean (WBM) operator.

Case 2 If $q=0$ and $r=0$, then the GWBM operator reduces to the following:

$$
\begin{aligned}
& \operatorname{GWBM}^{p, 0,0}\left(a_{1}, a_{2}, \ldots a_{n}\right)=\left(\sum_{i, j, k=1, i \neq j \neq k}^{n} w_{i} w_{j} w_{k} x_{i}^{p} x_{j}^{q}\right)^{\frac{1}{p}} \\
& =\left(\sum_{i=1}^{n} w_{i} x_{i}^{p} \sum_{j=1}^{n} w_{j} \sum_{k=1}^{n} w_{k}\right)^{\frac{1}{p}}
\end{aligned}
$$




$$
\operatorname{GWBM}^{p, 0,0}\left(a_{1}, a_{2}, \ldots a_{n}\right)=\left(\sum_{i=1}^{n} w_{i} x_{i}^{p}\right)^{\frac{1}{p}}
$$

which is the generalized weighted averaging operator. Further in this case, let us look at the GWBM operator for some special cases of $p$.

1. If $p=1$, the GWBM operator reduces to the weighted averaging (WA) operator.

2. If $p \rightarrow 0$, then the GWBM operator reduces to the weighted geometric (WG) operator.

3. If $p \rightarrow+\infty$, then the GWBM operator reduces to the max operator.

To aggregate the triangular fuzzy correlated information, based on the BM and weighted harmonic mean operators, H. Sun and M. Sun [23] developed the fuzzy Bonferroni harmonic mean operator. Since this operator considers the weight vector of the aggregated arguments, we redefine this operator as fuzzy weighted Bonferroni harmonic mean operator.

Definition 1.4 (42). Let $\widehat{a}_{i}=\left[a_{i}^{L}, a_{i}^{M}, a_{i}^{U}\right](i=1,2, \ldots n)$ be a collection of triangular fuzzy numbers, let $\left(w_{1}, w_{2}, \ldots w_{n}\right)$ be the weight vector $a_{i}(i=1,2, \ldots n)$ where $w_{i}>0, i=1,2, \ldots n$ and $\sum_{i=1}^{n} w_{i}=1$. If

$$
\operatorname{FWBHM}^{\mathrm{p}, \mathrm{q}}\left(\widehat{\mathrm{a}_{1}}, \hat{\mathrm{a}_{2}}, \ldots \widehat{\mathrm{a}_{\mathrm{n}}}\right)=\frac{1}{\left(\sum_{i, j=1}^{n}\left(w_{i} w_{j}\right) / \hat{\mathrm{a}}_{i}^{p} \hat{\mathrm{a}}_{j}^{q}\right)^{\frac{1}{p+q}}}
$$

$$
=\left[\frac{1}{\left(\sum_{i, j=1}^{n}\left(w_{i} w_{j}\right) /\left(\hat{a}_{i}^{L}\right)^{p}\left(\hat{a}_{j}^{L}\right)^{q}\right)^{\frac{1}{p+q}}}, \frac{1}{\left(\sum_{i, j=1}^{n}\left(w_{i} w_{j}\right) /\left(\hat{a}_{i}^{M}\right)^{p}\left(\hat{a}_{j}^{M}\right)^{q}\right)^{\frac{1}{p+q}}}, \frac{1}{\left(\sum_{i, j=1}^{n}\left(w_{i} w_{j}\right) /\left(\hat{a}_{i}^{U}\right)^{p}\left(\hat{a}_{j}^{U}\right)^{q}\right)^{\frac{1}{p+q}}}\right]
$$

where $p ; q \geq 0$, then $\mathrm{FWBHM}^{p, q}$ is called the fuzzy weighted Bonferroni harmonic mean (FWBHM) operator [10, 3].

In particular, considering the triangular fuzzy numbers. Let $\hat{a}_{i}=\left[a_{i}^{L}, a_{i}^{M}, a_{i}^{U}\right]$ $(i=1,2, \ldots n)$ reduce to the interval numbers $\hat{a}_{i}=\left[a_{i}^{L}, a_{i}^{M}\right](i=1,2, \ldots n)$ then the FWBHM operator (10) reduces to the uncertain weighted Bonferroni harmonic mean (UWBHM) operator as follows:

$$
\operatorname{UWBHM}^{\mathrm{p}, \mathrm{q}}\left(\widehat{a_{1}}, \hat{a_{2}}, \ldots \widehat{a_{n}}\right)=\frac{1}{\left(\sum_{i, j=1}^{n}\left(w_{i} w_{j}\right) / \hat{a}_{i}^{p} \hat{a}_{j}^{q}\right)^{\frac{1}{p+q}}}
$$




$$
=\left[\frac{1}{\left(\sum_{i, j=1}^{n}\left(w_{i} w_{j}\right) /\left(\hat{a}_{i}^{L}\right)^{p}\left(\hat{a}_{j}^{L}\right)^{q}\right)^{\frac{1}{p+q}}}, \frac{1}{\left(\sum_{i, j=1}^{n}\left(w_{i} w_{j}\right) /\left(\hat{a}_{i}^{U}\right)^{p}\left(\hat{a}_{j}^{U}\right)^{q}\right)^{\frac{1}{p+q}}}\right]
$$

If $w=(1 / n, 1 / n, \ldots 1 / n)^{T}$ then the UWBHM operator reduces to the uncertain Bonferroni harmonic mean (UBHM) operator as follows:

$$
\begin{gathered}
\operatorname{UBHM}^{\mathrm{p}, \mathrm{q}}\left(\mathrm{a}_{1}, \mathrm{a}_{2}, \ldots \mathrm{a}_{\mathrm{n}}\right)=\frac{1}{\left(1 / n^{2} \sum_{i, j=1}^{n}\left(w_{i} w_{j}\right) / \hat{a}_{i}^{p} \hat{a}_{j}^{q}\right)^{\frac{1}{p+q}}} \\
=\left[\frac{1}{\left(1 / n^{2} \sum_{i, j=1}^{n}\left(w_{i} w_{j}\right) /\left(\hat{a}_{i}^{L}\right)^{p}\left(\hat{a}_{j}^{L}\right)^{q}\right)^{\frac{1}{p+q}}}, \frac{1}{\left(1 / n^{2} \sum_{i, j=1}^{n}\left(w_{i} w_{j}\right) /\left(\hat{a}_{i}^{U}\right)^{p}\left(\hat{a}_{j}^{U}\right)^{q}\right)^{\frac{1}{p+q}}}\right]
\end{gathered}
$$

If $a_{i}^{L}=a_{i}^{U}=a_{i}$ for all, then the UBHM operator reduces to the weighted Bonferroni harmonic mean (WBHM) operator as follows:

$$
\operatorname{WBHM}^{\mathrm{p}, \mathrm{q}}\left(\mathrm{a}_{1}, \mathrm{a}_{2}, \ldots \mathrm{a}_{\mathrm{n}}\right)=\frac{1}{\left(\sum_{i, j=1}^{n}\left(w_{i} w_{j}\right) / a_{i}^{p} a_{j}^{q}\right)^{\frac{1}{p+q}}}
$$

In the Case $w=(1 / n, 1 / n, \ldots 1 / n)^{T}$ then the WBHM operator reduces to the Bonferroni harmonic mean (BHM) operator as follows:

$$
\operatorname{BHM}^{\mathrm{p}, \mathrm{q}}\left(\mathrm{a}_{1}, \mathrm{a}_{2}, \ldots \mathrm{a}_{\mathrm{n}}\right)=\frac{1}{\left(1 / n^{2} \sum_{i, j=1}^{n}\left(1 / a_{i}^{p} a_{j}^{q}\right)^{\frac{1}{p+q}}\right.}
$$

In recent years, the Schur convexity of functions relating to special means is a very significant research subject and has attracted the interest of many mathematicians. As supplements to the Schur convexity of functions, the Schur geometrically convex functions and Schur harmonically convex functions were investigated [8, 21, 26, 27]. 
In [9]. the authors discussed the Schur convexity, Schur geometric convexity, Schur harmonic convexity and Schur m-power convexity of the geometric Bonferroni mean.

This motivated us to determine the Schur convexity, Schur geometric convexity, Schur harmonic convexity and Schur m-power convexity of the Bonferroni harmonic mean.

Our main results are as follows.

Theorem 1.1 For fixed non-negative real numbers $p, q$ with $p+q \neq 0$, if $x=\left(x_{1}, x_{2}, \ldots x_{n}\right)$ then $\operatorname{BHM}^{p, q}(x)$ is Schur concave, Schur geometric convex and Schur harmonic convex on $R_{++}^{n}:=(0,+\infty)^{n}$.

Theorem 1.2 For fixed non-negative real numbers $p, q$ with $p+q \neq 0$, if $x=\left(x_{1}, x_{2}, \ldots x_{n}\right)$ then $(x)$ is Schur $m$ - power convexity on $R_{++}^{n}$.

1. If $m<0$, then $\mathrm{BHM}^{p, q, r}(X)$ is Schur m-power convex;

2. If $m>0$, then $\mathrm{BHM}^{p, q, r}(X)$ is Schur m-power concave;

3. If $m=0$, then $\mathrm{BHM}^{p, q, r}(X)$ is Schur m-power convex (concave);

\section{Preliminaries}

We begin with recalling some basic concepts and notations in the theory of majorization. For more details, we refer the reader to [2, 32].

Definition 2.1 Let $x=\left(x_{1}, x_{2}, x_{3} \ldots, x_{n}\right)$. and $y=\left(y_{1}, y_{2}, y_{3} \ldots, y_{n}\right) \in R^{n}$.

1. $x$ is said to be majorized by $y$ (in symbols $x \prec y$.), $\sum_{i=1}^{k} x_{[i]} \leq \sum_{i=1}^{k} y_{[i]}$ for $k=1,2,3, \ldots, n-1$. and $\sum_{\mathrm{i}=1}^{\mathrm{n}} \mathrm{X}_{\mathrm{i}}=\sum_{\mathrm{i}=1}^{\mathrm{n}} \mathrm{y}_{\mathrm{i}}$ where $x_{[1]} \geq, \ldots, \geq x_{[n]}$ and $y_{[1]} \geq \ldots \geq y_{[n]}$ are rearrangement of $x$ and $y$ and $y$ in a descending order.

2. $\Omega \subseteq R^{n}$ is called a convex set, if $\left(\alpha x_{1}+\beta y_{1}, \alpha x_{2}+\beta y_{2}, \ldots, \alpha x_{n}+\beta y_{n}\right) \in \Omega$, for any $x$ and $y \in \Omega$, where $\alpha$ and $\beta \in[0,1]$ with $\alpha+\beta=1$.

3. Let $\Omega \subseteq R^{n}$, the function $\varphi: \Omega \rightarrow R^{n}$ is said to be schur convex function on $\Omega$ if $x \prec y$ on $\Omega$ implies $\varphi(x) \leq \varphi(y)$. $\varphi$ is said to be a Schur concave function on $\Omega$, if and only if $-\varphi$ is Schur convex function.

Definition 2.2 [22] Let $x=\left(x_{1}, x_{2}, x_{3} \ldots, x_{n}\right)$. and $y=\left(y_{1}, y_{2}, y_{3}, \ldots, y_{n}\right) \in R_{+}^{n}$.

1. $\Omega \subseteq R^{n}$ is called geometrically convex set, if $\left(x_{1}^{\alpha} y_{1}^{\beta}, \ldots, x_{n}^{\alpha} y_{n}^{\beta}\right) \in R^{n}$ for any $\boldsymbol{x}$ and $y \in \Omega$, where $\alpha, \beta \in[0,1]$ with $\alpha+\beta=1$.

2. Let $\Omega \subseteq R_{+}^{n}$ the function $\varphi: \Omega \rightarrow R_{+}$is said to be schur geometrically convex function on $\Omega$ if $\left(\log \mathrm{x}_{1}, \log \mathrm{x}_{2} \ldots \log \mathrm{x}_{\mathrm{n}}\right)\left(\log \mathrm{y}_{1}, \log \mathrm{y}_{2} \ldots \log \mathrm{y}_{\mathrm{n}}\right)$ on $\Omega$ implies $\varphi(x) \leq \varphi(y) . \varphi$ is said to be a Schur geometrically concave function on $\Omega$ if and only if $-\varphi$ is Schur geometrically convex function. 
Definition 2.3 [4] Let $x=\left(x_{1}, x_{2}, x_{3} \ldots, x_{n}\right)$ and $y=\left(y_{1}, y_{2}, y_{3}, \ldots, y_{n}\right) \in R_{+}^{n}$.

1. A set $\Omega \subseteq R^{n}$. is said to be a harmonically convex set, if

$$
\left(\frac{x_{1} y_{1}}{\lambda x_{1}+(1-\lambda) y_{1}}, \frac{x_{2} y_{2}}{\lambda x_{2}+(1-\lambda) y_{2}}, \ldots, \frac{x_{n} y_{n}}{\lambda x_{n}+(1-\lambda) y_{n}}\right) \in \Omega
$$

for any $\boldsymbol{x}$ and $y \in \Omega$, and $\lambda \in[0,1]$.

2. A function $\varphi: \Omega \rightarrow R_{+}$. Is said to be a Schur-harmonically convex function on $\Omega$, if $\frac{1}{x} \prec \frac{1}{y}$ implies $\varphi(x) \leq \varphi(y) . \varphi$ is said to be a Schur harmonically concave function on $\Omega$. If and only if $-\varphi$ is a Schur-harmonically convex function.

Lemma 2.1 Let $\Omega \subseteq R^{n}$ be symmetric with non empty interior convex set and let $\varphi: \Omega \rightarrow R_{+}$be continuous on $\Omega$ and differentiable on $\Omega^{0}$. Then $\varphi$ is Schur convex (concave) if.

$$
\left(x_{1}-x_{2}\right)\left(\frac{\partial \phi(X)}{\partial x_{1}}-\frac{\partial \phi(X)}{\partial x_{2}}\right) \geq 0(\leq 0) \text {. }
$$

holds for any $x=\left(x_{1}, x_{2}, x_{3} \ldots, x_{n}\right) \in \Omega^{0}$.

Lemma 2.2 Let $\Omega \subseteq R^{n}$ be a symmetric geometrically convex set with non empty interior $\Omega^{0}$. Let $\varphi: \Omega \rightarrow R_{+}$be continuous on $\Omega$ and differentiable on $\Omega^{0}$. Then $\varphi$ is Schur gemetrically convex (concave) function $x=\left(x_{1}, x_{2}, x_{3} \ldots, x_{n}\right) \in \Omega^{0}$ if and only if $\phi$ is symmetric on $\Omega$ and

$$
\left(x_{1}-x_{2}\right)\left(x_{1} \frac{\partial \phi(X)}{\partial x_{1}}-x_{2} \frac{\partial \phi(X)}{\partial x_{2}}\right) \geq 0(\leq 0) .
$$

Lemma 2.3 Let $\Omega \subseteq R^{n}$ be symmetric harmonically convex set with non empty interior $\Omega^{0}$ Let $\varphi: \Omega \rightarrow R_{+}$be continuous on $\Omega$. and differentiable on $\Omega^{0}$ Then $\varphi$. is Schur harmonically convex (concave) function $x=\left(x_{1}, x_{2}, x_{3} \ldots, x_{n}\right) \in \Omega^{0}$. if and only if $\phi$ is symmetric on $\Omega$ and.

$$
\left(x_{1}-x_{2}\right)\left(x_{1}^{2} \frac{\partial \phi(X)}{\partial x_{1}}-x_{2}^{2} \frac{\partial \phi(X)}{\partial x_{2}}\right) \geq 0(\leq 0) .
$$

holds for any $x=\left(x_{1}, x_{2}, \ldots, x_{n}\right) \in \Omega^{0}$. 
Lemma 2.4 [20,29] Let $\varphi: \Omega \rightarrow R_{+}$be continuous on $\Omega$ and differentiable on $\Omega^{0}$. Then $\varphi$ is Schur m-power convex on function $x=\left(x_{1}, x_{2}, \ldots, x_{n}\right) \in \Omega^{0}$ if and only if $\varphi$ is symmetric on $\Omega$ and

$$
\frac{x_{1}^{m}-x_{2}^{m}}{m}\left[x_{1}^{m-1} \frac{\partial \varphi(x)}{\partial x_{1}}-x_{2}^{m-1} \frac{\partial \varphi(x)}{\partial x_{2}}\right] \geq 0 \text { if } m \neq 0
$$

and

$$
\left(\log x_{1}-\log x_{2}\right)\left[x_{1}^{m} \frac{\partial \varphi(x)}{\partial x_{1}}-x_{2}^{m} \frac{\partial \varphi(x)}{\partial x_{2}}\right] \geq 0 \text { if } m=0
$$

Lemma 2.5 Let $\left(x_{1}, x_{2}, \ldots x_{n},\right) \in R_{n}^{+}$and $A_{n}(x)=\frac{1}{n} \sum_{i=1}^{n} x_{i}$. Then

$$
u=\underbrace{A_{n}(x), A_{n}(x), \ldots \ldots A_{n}(x)}_{n} \prec\left(x_{1}, x_{2}, \ldots x_{n}\right)=x
$$

Lemma 2.6 If $x_{i}>0, i=1,2, \ldots n$, for any non negative constant $c$ satisfying $0 \leq c<\frac{1}{n} \sum_{i=1}^{n} x_{i}$ one has

$$
\left(\frac{x_{1}}{\sum_{i=1}^{n} x_{i}}, \ldots \frac{x_{n}}{\sum_{i=1}^{n} x_{i}}\right) \prec\left(\frac{x_{1}-c}{\sum_{i=1}^{n}\left(x_{i}-c\right)}, \ldots \frac{x_{n}-c}{\sum_{i=1}^{n}\left(x_{i}-c\right)}\right) .
$$

\section{Proof of main results}

The Bonferroni harmonic mean (BHM) is defined by

$$
\operatorname{BHM}^{\mathrm{p}, \mathrm{q}}(x)=\frac{1}{\left(1 / n^{2} \sum_{i, j=1}^{n}\left(1 / x_{i}^{p} x_{j}^{q}\right)^{\frac{1}{p+q}}\right.}
$$

Taking the natural logarithm gives

$$
\begin{gathered}
\log \mathrm{BHM}^{\mathrm{p}, \mathrm{q}}(x)=\log 1-\log \left(1 / n^{2} \sum_{i, j=1}^{n}\left(1 / x_{i}^{p} x_{j}^{q}\right)^{\frac{1}{p+q}}\right. \\
\log \mathrm{BHM}^{\mathrm{p}, \mathrm{q}}(x)=-\frac{1}{\mathrm{p}+\mathrm{q}}\left(\log n^{2}+\mathrm{Q}\right)
\end{gathered}
$$


Where

$$
\begin{aligned}
Q= & \sum_{J=3}^{n}\left[\log \left(\frac{1}{x_{1}^{p} x_{j}^{q}}\right)+\log \left(\frac{1}{x_{2}^{p} x_{j}^{q}}\right)\right]+\sum_{i=3}^{n}\left[\log \left(\frac{1}{x_{i}^{p} x_{1}^{q}}\right)+\log \left(\frac{1}{x_{i}^{p} x_{2}^{q}}\right)\right] \\
& +\left[\log \left(\frac{1}{x_{1}^{p} x_{2}^{q}}\right)+\log \left(\frac{1}{x_{2}^{p} x_{1}^{q}}\right)\right]+\sum_{i, j=3, i \neq j}^{n}\left[\log \left(\frac{1}{x_{i}^{p} x_{j}^{q}}\right)\right] .
\end{aligned}
$$

Partially differentiating the Eq. (3.1) with respect to $x_{1}$, we have

$$
\begin{aligned}
\frac{\partial \mathrm{BHM}^{\mathrm{p}, \mathrm{q}}(x)}{\partial x_{1}}= & \frac{\mathrm{BHM}^{\mathrm{p}, \mathrm{q}}(x)}{p+q} \frac{\partial}{\partial x_{1}} \sum_{J=3}^{n}\left[\log \left(\frac{1}{x_{1}^{p} x_{j}^{q}}\right)+\log \left(\frac{1}{x_{2}^{p} x_{j}^{q}}\right)\right] \\
& +\sum_{i=3}^{n}\left[\log \left(\frac{1}{x_{i}^{p} x_{1}^{q}}\right)+\log \left(\frac{1}{x_{i}^{p} x_{2}^{q}}\right)\right] \\
& +\left[\log \left(\frac{1}{x_{1}^{p} x_{2}^{q}}\right)+\log \left(\frac{1}{x_{2}^{p} x_{1}^{q}}\right)\right]
\end{aligned}
$$

$$
\begin{gathered}
\frac{\partial \mathrm{BHM}^{\mathrm{p}, \mathrm{q}}(x)}{\partial x_{1}}=\frac{(n-1) \mathrm{BHM}^{p, q}(x)}{p+q} \frac{\partial}{\partial x_{1}}\left[\log x_{1}^{p}+\log x_{1}^{q}\right] \\
\frac{\partial \mathrm{BHM}^{\mathrm{p}, \mathrm{q}}(x)}{\partial x_{1}}=\frac{(n-1) \mathrm{BHM}^{p, q}(x)}{p+q} \frac{p+q}{x_{1}}, \\
\frac{\partial \mathrm{BHM}^{\mathrm{p}, \mathrm{q}}(x)}{\partial x_{1}}=\frac{(n-1) \mathrm{BHM}^{p, q}(x)}{x_{1}} .
\end{gathered}
$$

Partially differentiating the Eq. (3.1) with respect to $x_{2}$, we have

$$
\begin{aligned}
\frac{\partial \mathrm{BHM}^{\mathrm{p}, \mathrm{q}}(x)}{\partial x_{2}}= & \frac{\mathrm{BHM}^{p, q}(x)}{p+q} \frac{\partial}{\partial x_{2}} \sum_{J=3}^{n}\left[\log \left(\frac{1}{x_{1}^{p} x_{j}^{q}}\right)+\log \left(\frac{1}{x_{2}^{p} x_{j}^{q}}\right)\right] \\
& +\sum_{i=3}^{n}\left[\log \left(\frac{1}{x_{i}^{p} x_{1}^{q}}\right)+\log \left(\frac{1}{x_{i}^{p} x_{2}^{q}}\right)\right] \\
& +\left[\log \left(\frac{1}{x_{1}^{p} x_{2}^{q}}\right)+\log \left(\frac{1}{x_{2}^{p} x_{1}^{q}}\right)\right]
\end{aligned}
$$




$$
\begin{gathered}
\frac{\partial \mathrm{BHM}^{p, q}(x)}{\partial x_{2}}=\frac{(n-1) \mathrm{BHM}^{p, q}(x)}{p+q} \frac{\partial}{\partial x_{2}}\left[\log x_{2}^{p}+\log x_{2}^{q}\right] \\
\frac{\partial \mathrm{BHM}^{p, q}(x)}{\partial x_{2}}=\frac{(n-1) \mathrm{BHM}^{p, q}(x)}{p+q} \frac{p+q}{x_{2}}, \\
\frac{\partial \mathrm{BHM}^{p, q}(x)}{\partial x_{2}}=\frac{(n-1) \mathrm{BHM}^{p, q}(x)}{x_{2}} .
\end{gathered}
$$

Proof of Theorem 1.1 By Lemma 2.1, direct computation gives

$$
\Delta_{1}=\left(x_{1}-x_{2}\right)\left(\frac{\partial \mathrm{BHM}^{p, q}(x)}{\partial x_{1}}-\frac{\partial \mathrm{BHM}^{p, q}(x)}{\partial x_{2}}\right)=\frac{-(n-1) \mathrm{BHM}^{p, q}\left(x_{1}-x_{2}\right)^{2}}{x_{1} x_{2}} \leq 0
$$

This implies that $\Delta_{1} \leq 0$ for $x \in R^{n}$ By Lemma 2.1, we conclude that BHM ${ }^{\mathrm{p}, \mathrm{q}}(\mathrm{x})$ is Schur concave on $R_{++}^{n}$.

In view of the discrimination criterion of Schur geometric convexity, we start with the following calculations:

$$
\Delta_{2}=\left(\log x_{1}-\log x_{2}\right)\left(x_{1} \frac{\partial \mathrm{BHM}^{p, q}(x)}{\partial x_{1}}-x_{2} \frac{\partial \mathrm{BHM}^{p, q}(x)}{\partial x_{2}}\right)=0 .
$$

This implies that $\Delta_{2}=0$ for $x \in R^{n}$

By Lemma 2.2, we conclude that $\mathrm{BHM}^{\mathrm{p}, \mathrm{q}}(\mathrm{x})$ is neither Schur geometrically convexity nor Schur geometrically concave on $R_{++}^{n}$.

Finally, we discuss the Schur harmonic convexity of $\mathrm{BHM}^{\mathrm{p}, \mathrm{q}}(\mathrm{x})$.

A direct computation gives

$$
\Delta_{3}=\left(x_{1}-x_{2}\right)\left(x_{1}^{2} \frac{\partial \mathrm{BHM}^{p, q}(x)}{\partial x_{1}}-x_{2}^{2} \frac{\partial \mathrm{BHM}^{p, q}(x)}{\partial x_{2}}\right)=(n-1) \operatorname{BHM}^{p, q}\left(x_{1}-x_{2}\right)^{2} \geq 0 \text {. }
$$

This implies that $\Delta_{3} \geq 0$ for $x \in R_{n}^{n}$. By Lemma 2.3, we conclude that BHM ${ }^{\mathrm{p}, \mathrm{q}}(\mathrm{x})$ is Schur harmonically convex on $R_{++}^{n}$.

This completes proof of Theorem 1.1 .

Proof of Theorem 1.2 Now we discuss the Schur m-power convexity of BHM ${ }^{\mathrm{p}, \mathrm{q}}(\mathrm{x})$.

It is easy to see that $\mathrm{BHM}^{\mathrm{p}, \mathrm{q}}(\mathrm{x})$ is symmetric on $R_{++}^{n}$. Without loss of generality, we may assume that $x_{1} \geq x_{2}$

A direct computation gives

$$
\Delta=\left(\frac{x_{1}^{m}-x_{2}^{m}}{m}\right)\left(x_{1}^{1-m} \frac{\partial \mathrm{BHM}^{p, q}(x)}{\partial x_{1}}-x_{2}^{1-m} \frac{\partial \mathrm{BHM}^{p, q}(x)}{\partial x_{2}}\right)
$$




$$
\begin{gathered}
=\frac{\left(x_{1}^{m}-x_{2}^{m}\right)(n-1) \mathrm{BHM}}{m}\left[\frac{1}{x_{1}^{m}}-\frac{1}{x_{2}^{m}}\right] \\
=\frac{-\left(x_{1}^{m}-x_{2}^{m}\right)^{2}(n-1) \mathrm{BHM}}{m x_{1}^{m} x_{2}^{m}} .
\end{gathered}
$$

If $m<0$, then $\Delta \geq 0$. From Lemma 2.4, it follows that BHM ${ }^{\mathrm{p}, \mathrm{q}}(\mathrm{x})$ is Schur $\mathrm{m}-$ power convex for $x \in R_{++}^{n}$.

If $\mathrm{m}>0$, then $\Delta \leq 0$. From Lemma 2.4, it follows that BHM ${ }^{\mathrm{p}, \mathrm{q}}(\mathrm{x})$ is Schur $\mathrm{m}-$ power convex for $x \in R_{++}^{n}$.

If $\mathrm{m}=0$, then by direct computation gives,

$$
\Delta=\left(\log x_{1}-\log x_{2}\right)\left(x_{1} \frac{\partial \mathrm{BHM}^{p, q}(x)}{\partial x_{1}}-x_{2} \frac{\partial \mathrm{BHM}^{p, q}(x)}{\partial x_{2}}\right)=0
$$

From Lemma 2.4, it follows that $\mathrm{BHM}^{\mathrm{p}, \mathrm{q}}(\mathrm{x})$ is neither Schur geometrically convexity nor Schur geometrically concave for $x \in R_{++}^{n}$.

The proof of Theorem 1.2 is completed.

\section{Applications}

Theorem 4.1 For fixed non-negative real numbers $p, q$ with $p+q \neq 0$, then for arbitrary $x \in R_{++}^{n}$.

$$
A_{n}(x) \geq \operatorname{BHM}^{p, q}(x)
$$

Proof From Theorem 1.2 $\mathrm{BHM}^{\mathrm{p}, \mathrm{q}}(\mathrm{x})$ is Schur concave on $R_{++}^{n}$.

Using Lemma 2.5, one has

$$
\underbrace{A_{n}(x), A_{n}(x), \ldots \ldots A_{n}(x)}_{n} \prec\left(x_{1}, x_{2}, . . x_{n}\right)
$$

Thus, we deduce from Definition

$2.1 \quad$ that $\mathrm{BHM}^{p, q}(x)\left(A_{n}(x), A_{n}(x), \ldots \ldots A_{n}(x)\right) \geq \operatorname{BHM}^{p, q}(x)\left(x_{1}, x_{2}, \ldots x_{n}\right)$

Which implies that

$$
A_{n}(x) \geq \operatorname{BHM}^{p, q}(x)
$$

Theorem 4.1 is proved. 
Theorem 4.2 For fixed non-negative real numbers $p, q$ with $p+q \neq 0$, and let $c$ be a constant satisfying $0 \leq c<A_{n}(x),(X-c)=\left(x_{1}-c, x_{2}-c, \ldots x_{n}-c\right)$ then for arbi$\operatorname{trary} x \in R_{++}^{n}$.

$$
\operatorname{BHM}^{p, q}(X-c) \leq\left(1-\frac{c}{A_{n}(x)}\right) \operatorname{BHM}^{p, q}(x)
$$

Proof By the majorization relationship given in Lemma (2.6),

$$
\left(\frac{x_{1}}{\sum_{i=1}^{n} x_{i}}, \ldots \frac{x_{n}}{\sum_{i=1}^{n} x_{n}}\right) \prec\left(\frac{x_{1}-c}{\sum_{i=1}^{n}\left(x_{i}-c\right)}, \ldots \frac{x_{n}-c}{\sum_{i=1}^{n}\left(x_{n}-c\right)}\right),
$$

From Theorem (1.1)

$$
\mathrm{BHM}^{p, q}\left(\frac{x_{1}}{\sum_{i=1}^{n} x_{i}}, \ldots \frac{x_{n}}{\sum_{i=1}^{n} x_{n}}\right) \geq \operatorname{BHM}^{p, q}\left(\frac{x_{1}-c}{\sum_{i=1}^{n}\left(x_{i}-c\right)}, \ldots \frac{x_{n}-c}{\sum_{i=1}^{n}\left(x_{n}-c\right)}\right)
$$

i.e.,

$$
\frac{\operatorname{BHM}^{p, q}\left(x_{1}, x_{2}, \ldots x_{n}\right)}{\sum_{i=1}^{n} x_{i}} \geq \frac{\operatorname{BHM}^{p, q}\left(x_{1}-c, x_{2}-\mathrm{c} \ldots x_{n}-c\right)}{\sum_{i=1}^{n} x_{i}-n c}
$$

which implies that

$$
\operatorname{BHM}^{p, q}(X-c) \leq\left(1-\frac{c}{A_{n}(x)}\right) \operatorname{BHM}^{p, q}(x)
$$

Theorem 4.2 is proved.

\section{Conclusion}

We prove the Bonferroni mean $\mathrm{BHM}^{p, q}$ by introducing non-negative parameters $p, q$ under the condition of Schur concave, Schur geometric convex and Schur harmonic convex on $R_{++}^{n}$.

As an application of the Schur convexity, we establish two inequalities for generalized geometric Bonferroni mean $\mathrm{BHM}^{p, q}$. For details, we refer the interested reader to $[15,16,24,28,33,34]$ and the references therein 
Author contributions All authors contributed equally and significantly in this paper. All authors read and approved the final manuscript.

\section{Compliance with ethical standards}

Conflict of interest The authors declare that they have no conflict of interest.

Open Access This article is distributed under the terms of the Creative Commons Attribution 4.0 International License (http://creativecommons.org/licenses/by/4.0/), which permits unrestricted use, distribution, and reproduction in any medium, provided you give appropriate credit to the original author(s) and the source, provide a link to the Creative Commons license, and indicate if changes were made.

\section{References}

1. Beliakov, G., S. James, J. Mordelov, T. Rckschlossov, and R.R. Yager. 2010. Generalized Bonferroni mean operators in multicriteria aggregation. Fuzzy Sets and Systems 161 (17): 2227-2242.

2. Bonferroni, C. 1950. Sulle medie multiple di potenze. Bollettino dell Unione Matematica Italiana 5 (3-4): 267-270.

3. Chiclana, F., F. Herrera, and E. Herrera-Viedma. 2001. Integrating multiplicative preference relations in a multipurpose decisionmaking model based on fuzzy preference relations. Fuzzy Sets and Systems 122 (2): 277-291.

4. Chu, Y., and Y. Lv. 2009. The Schur harmonic convexity of the Hamy symmetric function and its applications. Journal of Inequalities and Applications. 2009: 838529.

5. Chu, Y.M., and W.F. Xia. 2010. Necessary and su_cient conditions for the Schur harmonic convexity of the gener-alized Muirhead mean. Proc. A. Razmadze Math. Inst. 152: 19-27.

6. Chu, Y., X.M. Zhang, and G.D. Wang. 2008. The Schur geometrical convexity of the extended mean values. Journal of Convex Analysis 15 (4): 707-718.

7. Chu, Y.M., G.D. Wang, and X.H. Zhang. 2011. The Schur multiplicative and harmonic convexities of the complete symmetric function. Mathematische Nachrichten 284 (5-6): 653-663.

8. Fu, L.L., B.Y. Xi, and H.M. Srivastava. 2011. Schur-convexity of the generalized Heronian means involving two positive numbers. Taiwanese Journal of Mathematics 15 (6): 2721-2731.

9. Huan -Nan Shi. 2018. Shan-He Wu Schur convexity of the generalized geometric Bonferroni mean and the relevant inequalities. Journal of Inequalities and Applications 2018: 8.

10. van Laarhoven, P.J.M., and W. Pedrycz. 1983. A fuzzy extension of Saatys priority theory. Fuzzy Sets and Systems 11 (3): 229-241.

11. Marshall, A.W., I. Olkin, and B.C. Arnold. 2011. Inequalities: theory of majorization and its application, vol. 2nd. New York: Springer.

12. Meng, J.X., Y.M. Chu, and X.M. Tang. 2010. The Schur-harmonic-convexity of dual form of the Hamy symmetric function. Matematički vesnik. 62 (1): 37-46.

13. Park, JH, Park, EJ. (2013) Generalized fuzzy Bonferroni harmonic mean operators and their applications in group decision making. Journal of Applied Mathematics 2013:604029. https://doi. org/10.1155/2013/604029.

14. Park, J.H., and J.Y. Kim. 2016. Intuitionistic fuzzy optimized weighted geometric Bonferroni means and their applications in group decision making. Fundamenta Informaticae 144 (3-4): 363-381.

15. Qi, F. 2005. A note on Schur-convexity of extended mean values. Rocky Mountain Journal of Mathematics 35 (5): 1787-1793.

16. Qi, F., J. Sndor, S.S. Dragomir, and A. Sofo. 2005. Notes on the Schur-convexity of the extended mean values. Taiwanese Journal of Mathematics 9 (3): 411-420.

17. Shi, H.N. 2012. Theory of majorization and analytic inequalities. Harbin: Harbin Institute of Technology Press. (in Chinese).

18. Shi, H.N., and J. Zhang. 2013. Some new judgement theorems of Schur geometric and Schur harmonic convexities for a class of symmetric functions. Journal of Inequalities and Applications 2013: 527. 
19. Shi, H.N., and J. Zhang. 2014. Schur-convexity, Schur geometric and Schur harmonic convexities of dual form of a class symmetric functions. J. Math. Inequal. 8 (2): 349-358.

20. Shi, H.N., and S.H. Wu. 2017. Schur m-power convexity of geometric Bonferroni mean. Italian Journal of Pure and Applied Mathematics 38: 769-776.

21. Shi, H.N., S.H. Wu, and F. Qi. 2006. An alternative note on the Schur-convexity of the extended mean values. Math. Inequal. Appl. 9: 219-224.

22. Shi, H.N., J. Zhang, and Q.H. Ma. 2016. Schur-convexity, Schur-geometric and Schur-harmonic convexity for a composite function of complete symmetric function. Springer Plus 5: 296.

23. Sun, H., and M. Sun. 2012. Generalized Bonferroni harmonic mean operators and their application to multiple attribute decision making. Journal of Computational Information Systems 8 (14): $5717-5724$

24. Sun, J., Z.L. Sun, B.Y. Xi, and F. Qi. 2015. Schur-geometric and Schur-harmonic convexity of an integral mean for convex functions. Turk. J. Anal. Number Theory 3 (3): 87-89.

25. Wei, G.-W. 2011. FIOWHM operator and its application to multiple attribute group decision making. Expert Systems with Applications 38 (4): 2984-2989.

26. Wu, S.H. 2005. Generalization and sharpness of power means inequality and their applications. Journal of Mathematical Analysis and Applications 312: 637-652.

27. Wu, S.H., and H.N. Shi. 2011. A relation of weak majorization and its applications to certain inequalities for means. Mathematica Slovaca 61 (4): 561-570.

28. $\mathrm{Wu}, \mathrm{Y}$., and F. Qi. 2012. Schur-harmonic convexity for differences of some means. Analysis 32 (4): 263-270.

29. Xia, M., Z. Xu, and B. Zhu. 2012. Generalized intuitionistic fuzzy Bonferroni means. International Journal of Intelligent Systems 27 (1): 23-47.

30. Xu, Z. 2009. Fuzzy harmonic mean operators. International Journal of Intelligent Systems 24 (2): $152-172$.

31. Xu, Z., and R.R. Yager. 2011. Intuitionistic fuzzy Bonferroni means. IEEE Transactions on Systems, Man, and Cybernetics 41 (2): 568-578.

32. Yager, R.R. 2009. On generalized Bonferroni mean operators for multi-criteria aggregation. International Journal of Approximate Reasoning 50 (8): 1279-1286.

33. Yang, Z.H. 2012. Schur power convexity of Stolarsky means. Publicationes Mathematicae (Debrecen) 80 (1-2): 43-66.

34. Yang, Z.H. 2013. Schur power convexity of the Darczy means. Math. Inequal. Appl. 16 (3): $751-762$.

35. Zhang, X.M., and Z.H. Yang. 2007. Differential criterion of n-dimensional geometrically convex functions. Journal of Applied Analysis 13 (2): 197-208.

36. Zheng, N.G., Z.H. Zhang, and X.M. Zhang. 2007. Schur-convexity of two types of one-parameter mean values in $\mathrm{n}$ variables. Journal of Inequalities and Applications 2007: 078175. 